\title{
Synergistic image reconstruction for hybrid ultrasound and photoacoustic computed tomography
}

Thomas P. Matthews, Kun Wang, Lihong V. Wang, Mark A. Anastasio

Thomas P. Matthews, Kun Wang, Lihong V. Wang, Mark A. Anastasio, "Synergistic image reconstruction for hybrid ultrasound and photoacoustic computed tomography," Proc. SPIE 9323, Photons Plus Ultrasound: Imaging and Sensing 2015, 93233A (11 March 2015); doi: 10.1117/12.2081048

SPIE. Event: SPIE BiOS, 2015, San Francisco, California, United States 


\title{
Synergistic Image Reconstruction for Hybrid Ultrasound and Photoacoustic Computed Tomography
}

\author{
Thomas P. Matthews ${ }^{a}$, Kun Wang ${ }^{a}$, Lihong V. Wang ${ }^{a}$, and Mark A. Anastasio ${ }^{a}$ \\ ${ }^{a}$ Department of Biomedical Engineering, School of Engineering and Applied Science, \\ Washington University in St. Louis, St. Louis, MO 63130
}

\begin{abstract}
Conventional photoacoustic computed tomography (PACT) image reconstruction methods assume that the object and surrounding medium are described by a constant speed-of-sound (SOS) value. In order to accurately recover fine structures, SOS heterogeneities should be quantified and compensated for during PACT reconstruction. To address this problem, several groups have proposed hybrid systems that combine PACT with ultrasound computed tomography (USCT). In such systems, a SOS map is reconstructed first via USCT. Consequently, this SOS map is employed to inform the PACT reconstruction method. Additionally, the SOS map can provide structural information regarding tissue, which is complementary to the functional information from the PACT image. We propose a paradigm shift in the way that images are reconstructed in hybrid PACT-USCT imaging. Inspired by our observation that information about the SOS distribution is encoded in PACT measurements, we propose to jointly reconstruct the absorbed optical energy density and SOS distributions from a combined set of USCT and PACT measurements, thereby reducing the two reconstruction problems into one. This innovative approach has several advantages over conventional approaches in which PACT and USCT images are reconstructed independently: (1) Variations in the SOS will automatically be accounted for, optimizing PACT image quality; (2) The reconstructed PACT and USCT images will possess minimal systematic artifacts because errors in the imaging models will be optimally balanced during the joint reconstruction; (3) Due to the exploitation of information regarding the SOS distribution in the full-view PACT data, our approach will permit high-resolution reconstruction of the SOS distribution from sparse array data.
\end{abstract}

Keywords: ultrasound computed tomography, photoacoustic computed tomography, joint image reconstruction

\section{INTRODUCTION}

Ultrasound computed tomography (USCT), which measures the speed-of-speed (SOS), and photoacoustic computed tomography (PACT), which measures absorbed optical energy density, are two imaging modalities that have a wide range of potential applications from pre-clinical small animal imaging to large-scale screening of human subjects. ${ }^{1-7}$ Their complementary contrasts and similar implementations have led several groups to develop hybrid USCT/PACT imaging systems. ${ }^{5,8}$ The strategy of how to best reconstruct images from these systems thus remains an important question. ${ }^{9,10}$ Most PACT image reconstruction algorithms assume a constant SOS distribution. ${ }^{11}$ For many biological applications, this is a poor assumption that can result in reduced resolution, reduced contrast, and an increase in the number of imaging artifacts. ${ }^{9,11}$ In addition to providing structural information complementary to the functional contrast of PACT, USCT can be used to estimate the SOS map.

In cases where both USCT and PACT data are available, the conventional image reconstruction approach is to first reconstruct a SOS map from the USCT measurements and then reconstruct the absorbed optical energy density from the PACT data using this estimate. However, reconstructing the SOS and absorbed optical energy density independently is not optimal. Since the propagation of photoacoustic waves depends on the acoustic properties of the underlying medium, information on the SOS distribution is encoded in the PACT measurements. By reconstructing the images independently, this information is wasted.

Further author information: (Send correspondence to: Mark A. Anastasio)

Mark A. Anastio: E-mail: anastasio@wustl.edu

Photons Plus Ultrasound: Imaging and Sensing 2015, edited by Alexander A. Oraevsky, Lihong V. Wang

Proc. of SPIE Vol. 9323, 93233A - C) 2015 SPIE · CCC code: $1605-7422 / 15 / \$ 18$

doi: $10.1117 / 12.2081048$

Proc. of SPIE Vol. 9323 93233A-1 
One method to exploit this information is by joint reconstruction of the SOS and absorbed optical energy density from a combined set of USCT and PACT measurements. This approach has several advantages over conventional approaches. First, variations in the SOS distribution are automatically accounted for when reconstructing the absorbed optical energy density image. Second, errors in the imaging models will be balanced during the joint reconstruction, which may lead to a reduction in the number of imaging artifacts. Finally, the addition of the acoustic information from the PACT measurements will reduce the number of USCT measurements needed to reconstruct an accurate SOS map. This could simplify hardware designs by requiring fewer ultrasonic transducers to be able to transmit and reduce image acquisition times.

\section{BACKGROUND}

\subsection{Photoacoustic computed tomography}

In PACT, an object of interest is illuminated with a laser pulse, resulting in the generation of a photoacoustic wave, which is then measured by a collection of ultrasonic transducers surrounding the object. This can be described, mathematically, in operator form as

$$
\mathbf{g}_{p a}=\mathcal{M} \mathbf{H}_{p a}^{\mathbf{c}} \mathbf{A}
$$

where $\mathbf{A}$ is the absorbed optical energy density, $\mathbf{g}_{p a}$ is the measured photoacoustic data, $\mathbf{H}_{p a}^{\mathbf{c}}$ is an operator that computes the action of the photoacoustic wave equation, and $\mathcal{M}$ is a sampling matrix that describes the act of measurement by the transducers. The superscript $\mathbf{c}$ in $\mathbf{H}_{p a}^{\mathbf{c}}$ is used to emphasize that this operator depends on the SOS c.

Using this, an estimate of the absorbed optical energy density can then be found by solving the following optimization problem,

$$
\hat{\mathbf{A}}=\underset{\mathbf{A} \geq 0}{\arg \min } \frac{1}{2}\left\|\underline{\mathbf{g}_{p a}}-\mathcal{M} \mathbf{H}_{p a}^{\mathbf{c}} \mathbf{A}\right\|_{2}^{2}+\mu \Phi(\mathbf{A})
$$

where $\mathbf{g}_{p a}$ is the recorded pressure data, $\Phi$ is a regularization function, and $\mu$ is the corresponding regularization parameter. Many optimization methods require calculation of the gradient of the cost function with respect to the variable of interest. The gradient with respect to $\mathbf{A}$ can be calculated via the method described by Huang et al. ${ }^{11}$ Then, the optimization can be solved using any of a number of methods, including gradient descent and FISTA. $^{12}$

In general, the SOS distribution is not known. One approach to overcome this is to attempt to jointly estimate $\mathbf{A}$ and $\mathbf{c}$ from the PACT measurements alone using the following optimization problem,

$$
\hat{\mathbf{A}}, \hat{\mathbf{c}}=\underset{\mathbf{A} \geq 0, \mathbf{c} \in \mathcal{C}}{\arg \min } \frac{1}{2}\left\|\mathbf{g}_{p a}-\mathcal{M} \mathbf{H}_{p a}^{\mathbf{c}} \mathbf{A}\right\|_{2}^{2}+\mu \Phi(\mathbf{A})+\lambda \Psi(\mathbf{c}),
$$

where $\mathcal{C}$ is a convex set, $\Psi$ is a regularization function, and $\lambda$ is its regularization parameter. This problem can be solved using an alternating minimization strategy. In this technique, the problem is divided into two subproblems, one in which $\mathbf{A}$ is estimated for a fixed $\mathbf{c}$ and one in which $\mathbf{c}$ is estimated for a fixed $\mathbf{A}$. This approach is described in more detail by Huang et al. ${ }^{13}$ While this strategy can be effective in some cases, the problem suffers from numerical instabilities, and often it may not be possible to accurately reconstruct both $\mathbf{A}$ and $\mathbf{c .}^{13,14}$

\subsection{Ultrasound computed tomography}

In USCT, a collection of ultrasonic transducers surround the object of interest. In turn, each transducer emits an ultrasonic pulse. The resulting pressure wave propagates through the object and is then measured by all transducers. This can be described, mathematically, in operator form as

$$
\mathbf{g}_{m}=\mathcal{M} \mathbf{H}_{u s}^{\mathbf{c}} \mathbf{s}_{m}
$$


where the index $m$ denotes the $m$-th view (out of $M$ ), $\mathbf{g}_{m}$ is the measured pressure data, $\mathbf{s}_{m}$ is the known excitation pulse, and $\mathbf{H}_{u s}^{\mathbf{c}}$ is the operator that denotes the action of the wave equation. Note that $\mathbf{H}_{p a}^{\mathbf{c}}$ and $\mathbf{H}_{u s}^{\mathbf{c}}$ are identical aside from the source terms and initial conditions in the wave equation.

An estimate of the SOS is obtained by solving the following optimization problem

$$
\hat{\mathbf{c}}=\arg \min _{\mathbf{c} \in \mathcal{C}} \frac{1}{2} \sum_{m=0}^{M-1}\left\|\underline{\mathbf{g}_{m}}-\mathcal{M} \mathbf{H}_{u s}^{\mathbf{c}} \mathbf{s}_{m}\right\|_{2}^{2}+\lambda \Psi(\mathbf{c}),
$$

where $\mathbf{g}_{m}$ is the recorded pressure data for the $m$-th view. When $M$ is large, evaluating this objective function is computationally expensive. A source encoding technique can be employed to obtain an equivalent optimization problem whose solution can be computed more efficiently, ${ }^{15-20}$

$$
\hat{\mathbf{c}}=\arg \min _{\mathbf{c} \in \mathcal{C}} \mathbf{E}_{\mathbf{w}}\left[\frac{1}{2}\left\|\underline{\mathbf{g}}^{\mathbf{w}}-\mathcal{M} \mathbf{H}_{u s}^{\mathbf{c}} \mathbf{s}^{\mathbf{w}}\right\|_{2}^{2}\right]+\lambda \Psi(\mathbf{c}),
$$

where $\mathbf{w}$ is a random encoding vector with zero mean and identity covariance matrix, $\mathbf{E}_{\mathbf{w}}$ is the expectation with respect to $\mathbf{w}$, and

$$
\underline{\mathbf{g}}^{\mathrm{w}}=\sum_{m=0}^{M-1}[\mathbf{w}]_{m} \underline{\mathbf{g}_{m}} \quad \text { and } \quad \mathbf{s}^{\mathbf{w}}=\sum_{m=0}^{M-1}[\mathbf{w}]_{m} \mathbf{s}_{m}
$$

are the encoded recorded pressure data and the encoded source term respectively. The gradient of the objective function in Eqn. 6 can be calculated using the adjoint state method ${ }^{21}$ and the optimization problem solved using stochastic gradient descent. ${ }^{20}$

\section{SYNERGISTIC IMAGE RECONSTRUCTION}

By combining USCT and PACT measurements, the joint optimization problem can be written as

$$
\hat{\mathbf{A}}, \hat{\mathbf{c}}=\underset{\mathbf{A} \geq 0, \mathbf{c} \in \mathcal{C}}{\arg \min } \frac{1}{2}\left\|\mathbf{g}_{p a}-\mathcal{M} \mathbf{H}_{p a}^{\mathbf{c}} \mathbf{A}\right\|_{2}^{2}+\mathbf{E}_{\mathbf{w}}\left[\frac{1}{2}\left\|\underline{\mathbf{g}}^{\mathbf{w}}-\mathcal{M} \mathbf{H}_{u s}^{\mathbf{c}} \mathbf{s}^{\mathbf{w}}\right\|_{2}^{2}\right]+\lambda \Psi(\mathbf{c})+\mu \Phi(\mathbf{A}) .
$$

This optimization problem can likewise be solved using an alternating minimization approach. In this case, the subproblem to estimate A remains the same, but the subproblem to estimate $\mathbf{c}$ will now include both the PACT and USCT measurements. Its formulation is given below.

$$
\hat{\mathbf{c}}=\underset{\mathbf{c} \in \mathcal{C}}{\arg \min } \frac{1}{2}\left\|\mathbf{g}_{p a}-\mathcal{M} \mathbf{H}_{p a}^{\mathbf{c}} \mathbf{A}\right\|_{2}^{2}+\mathbf{E}_{\mathbf{w}}\left[\frac{1}{2}\left\|\underline{\mathbf{g}}^{\mathbf{w}}-\mathcal{M} \mathbf{H}_{u s}^{\mathbf{c}} \mathbf{s}^{\mathbf{w}}\right\|_{2}^{2}\right]+\lambda \Psi(\mathbf{c}) .
$$

This alternating minimization approach is summarized in Algorithm 1.

\section{DESCRIPTION OF COMPUTER-SIMULATION STUDIES}

\subsection{Generation of pressure data}

Numerical phantoms were generated for the absorbed optical energy density and the SOS, as shown in Fig. 1. The pressure was simulated on a Cartesian grid with $256 \times 256$ pixels and a pixel size of $0.5 \mathrm{~mm}$. A square transducer array with 800 evenly-distributed elements was employed. The length of one side of the array was $100 \mathrm{~mm}$. The pressure was recorded for 2000 time samples with a time step of $75 \mathrm{~ns}$. For the PACT dataset, an initial pressure distribution corresponding in shape to the absorbed optical energy density phantom was employed. The pressure was recorded by all 800 transducers. The propagation of the acoustic and photoacoustic wavefields were simulated using a wave equation solver based on the first-order pseudospectral k-space method. ${ }^{22,23}$ For the USCT dataset used in the joint reconstruction, pressure data was simulated for 8, 16, 40, and 100 views. For each view, one of the transducers served as the emitter, while all 800 transducers recorded the pressure. The emitters were chosen to be evenly distribution among the 800 transducers. No noise was added to the pressure data. 


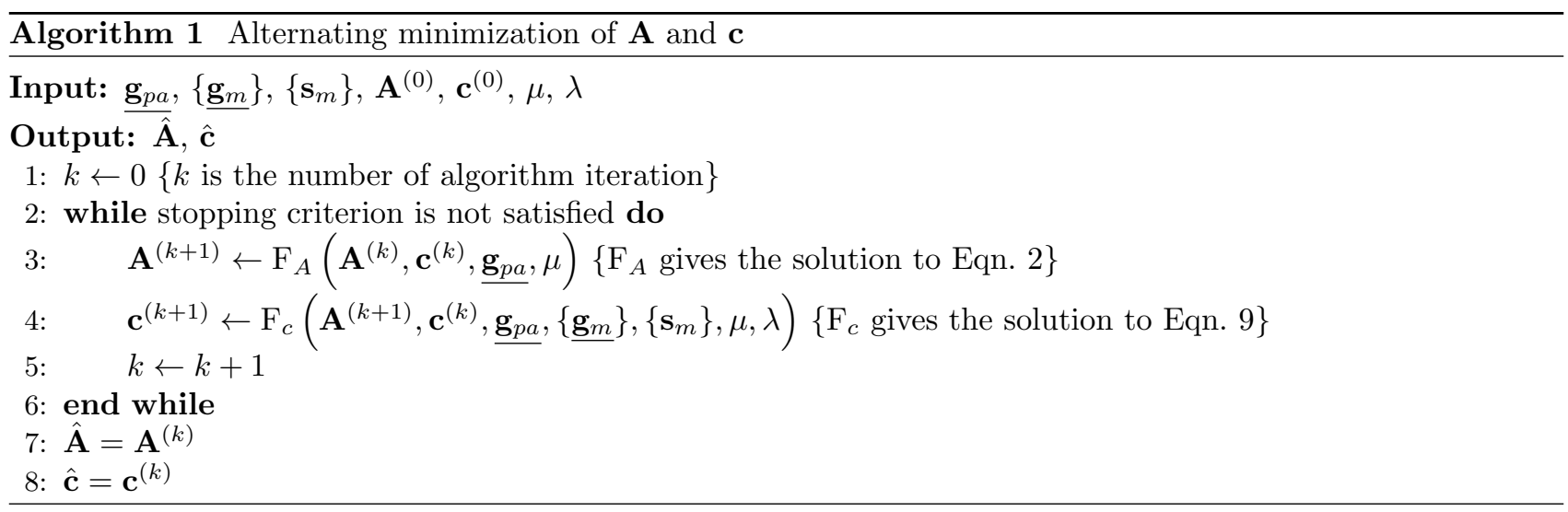

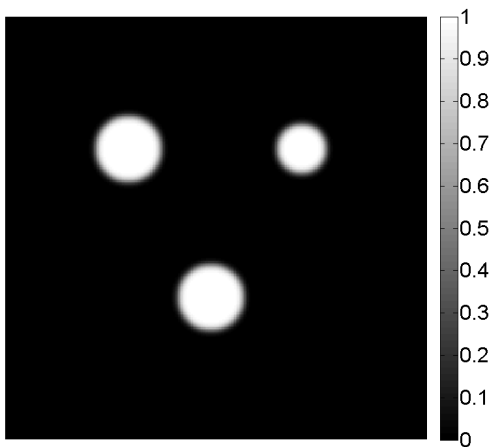

(a)

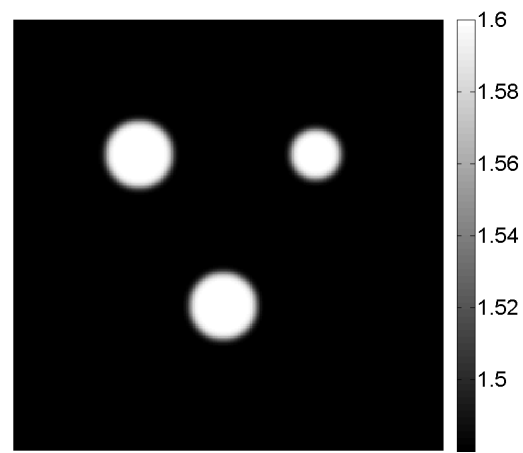

(b)

Figure 1: Numerical phantoms for (a) absorbed optical energy density and (b) speed-of-sound $[\mathrm{mm} / \mu \mathrm{s}]$.

\subsection{Image reconstruction}

In this preliminary study, the number of pixels, the pixel size, the number of time steps, and the time interval were all the same for the joint reconstruction results as for the pressure generation. The subproblem for estimating A was solved using FISTA with a fixed step size of 0.01. The subproblem for estimating $\mathbf{c}$ was solved using gradient descent with a backtracking line search. A was constrained to be positive, while $\mathbf{c}$ was forced to be within $[1.0,3.0] \mathrm{mm} / \mu \mathrm{s}$. Unless otherwise noted, the initial guess for $\mathbf{A}$ was chosen to be all zeros and the initial guess for $\mathbf{c}$ was chosen to be the background SOS. The stopping criterion for the subproblem estimating $\mathbf{A}$ was when the relative change in the cost function between subsequent iterations was less than $10^{-3}$. The subproblem estimating $\mathbf{c}$ was stopped after a fixed number of iterations (20). For the joint reconstruction using both PACT and USCT measurements, the gradients with respect to $\mathbf{c}$ for the PACT data fidelity term and for the USCT data fidelity term were each normalized by their $\ell_{2}$-norms. For the source-encoding study, the sound speed was reconstructed using a second-order k-space method. ${ }^{24}$ In this case, the simulation grid contained $1024 \times 1024$ pixels due to the lack of a perfectly matched layer. The regularization functions for both $\mathbf{A}$ and $\mathbf{c}$ were taken to be a total variation penalty, defined as

$$
\|\mathbf{x}\|_{T V}=\sum_{i=1}^{N_{x}-1} \sum_{j=1}^{N_{y}-1} \sqrt{\epsilon+\left([x]_{j N_{x}+i}-[x]_{j N_{x}+i-1}\right)^{2}+\left([x]_{j N_{x}+i}-[x]_{(j-1) N_{x}+i}\right)^{2}},
$$

where $N_{x}$ and $N_{y}$ are the dimensions of the reconstructed image, and $\epsilon$ was a small constant empirically chosen to be $10^{-12}$ used to avoid dividing by zero when calculating the gradient.

\section{NUMERICAL RESULTS}

The images obtained via joint reconstruction using PACT data alone are shown in Fig. 2. While $\mathbf{A}$ is accurately estimated, the estimate of $\mathbf{c}$ contains obvious artifacts. This is confirmed by comparing the profiles through the 


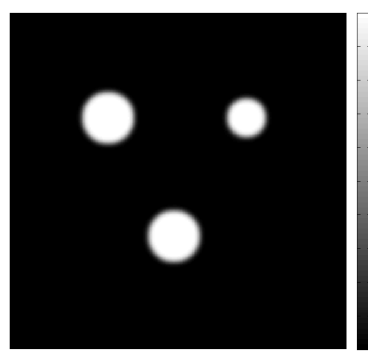

(a)

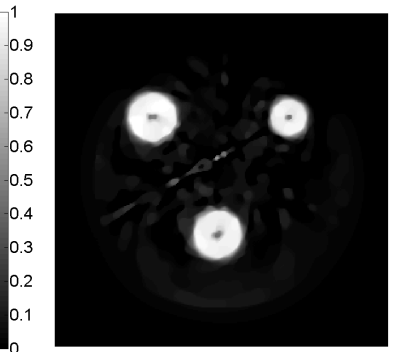

(b)

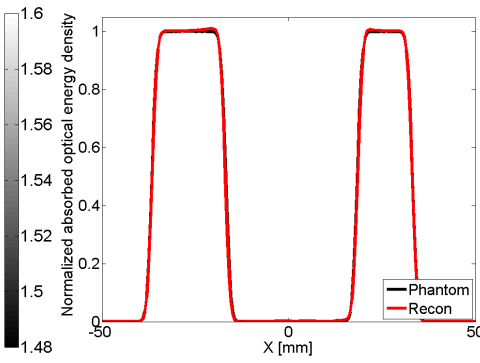

(c)

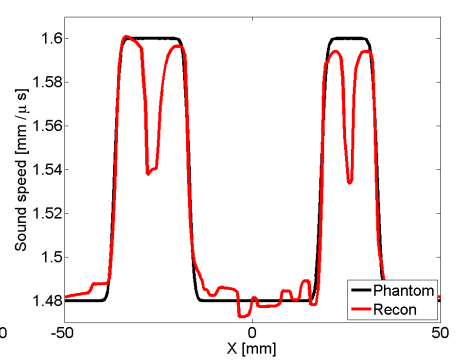

(d)

Figure 2: Joint reconstruction of (a) absorbed optical energy density and (b) speed-of-sound $[\mathrm{mm} / \mu \mathrm{s}]$ from PACT data alone. Profiles through the centers of the reconstructed (c) absorbed optical energy density and (d) speed-of-sound images. The profiles of the phantom are shown in black and the profiles of the reconstructed images are in red.

centers of the reconstructed images with the profiles of the true phantoms. These images were obtained after 36 iterations using regularization parameter values of $10^{-4}$ and $10^{-3}$ for $\mu$ and $\lambda$, respectively.

An alternative strategy to joint reconstruction is to first reconstruct $\mathbf{c}$ from a collection of USCT measurements and then use this estimate when reconstructing $\mathbf{A}$. However, this approach fails to exploit the acoustic information in the PACT data. Thus, it may require more views to accurately reconstruct $\mathbf{c}$ than joint reconstruction using combined PACT and USCT data. Figure 3 shows the reconstructed SOS for different numbers of views (i.e. different number of emitters). The images were reconstructed using the source encoding method described by Eqn. 6 and are shown after 300 iterations. A regularization parameter value of $10^{-3}$ was used. As expected, when the number of views is decreased, the accuracy of the reconstructed SOS is reduced. This is seen in the profiles through the object.

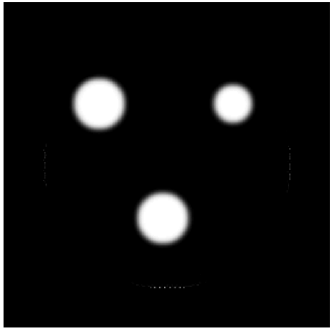

(a)

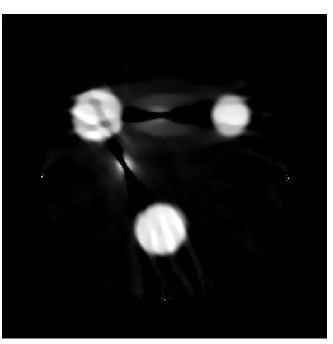

(c)

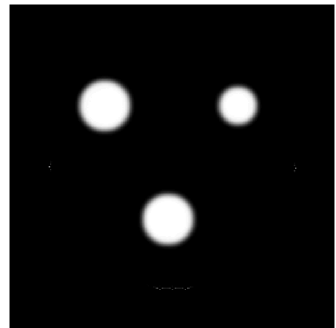

(b)

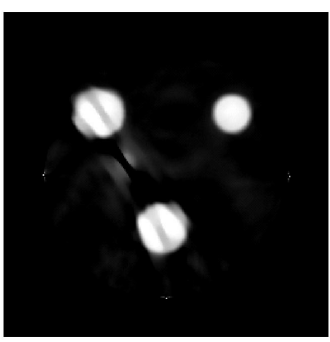

(d)

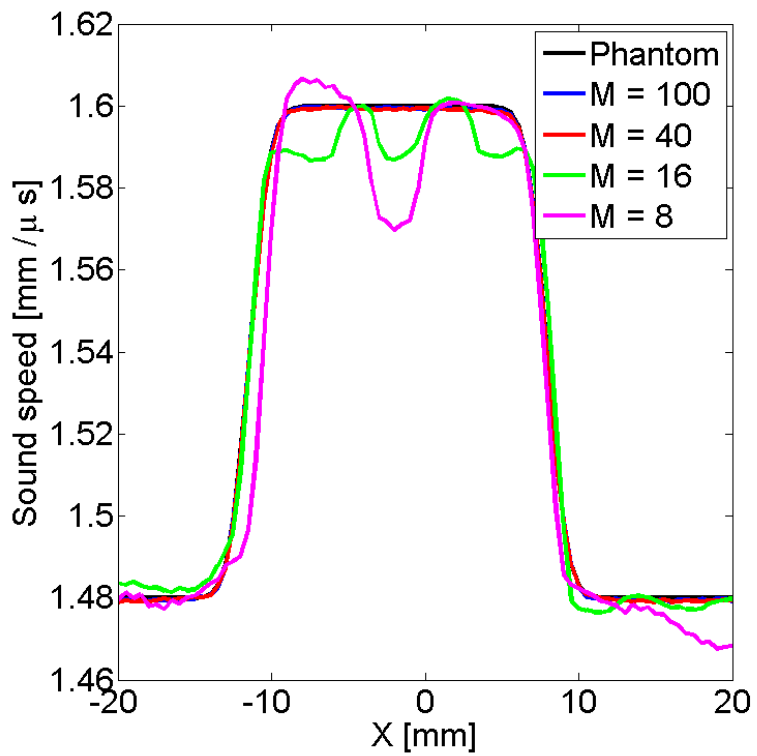

(e)

Figure 3: Reconstruction of the SOS from USCT measurements using (a) 100 views, (b) 40 views, (c) 16 views, and (d) 8 views. The grayscale window is $[1.481 .60] \mathrm{mm} / \mu \mathrm{s}$. (e) Profiles through the center of the images.

From these results, reconstruction of the SOS from a sparse array of USCT measurements appears challenging. However, when joint reconstruction using the combined USCT and PACT data is performed, an accurate estimate 


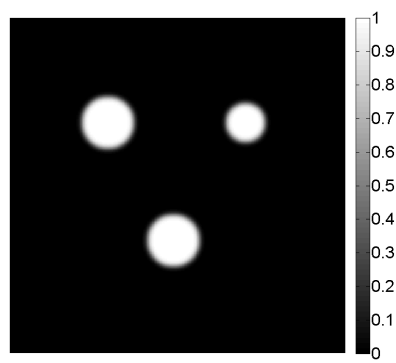

(a)

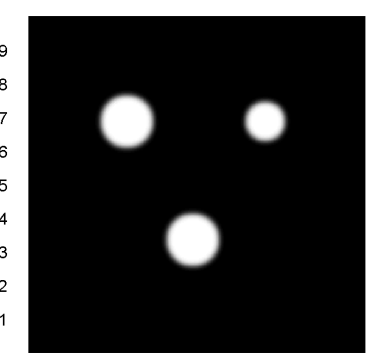

(b)

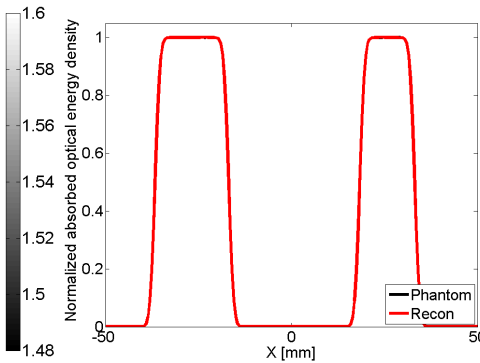

(c)

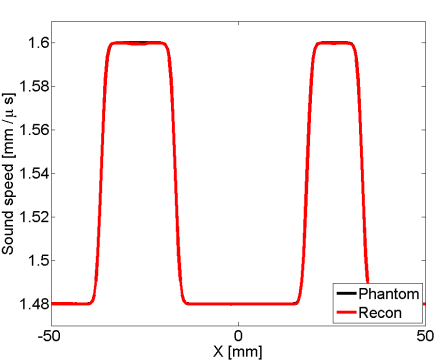

(d)

Figure 4: Synergistic reconstruction of (a) absorbed optical energy density and (b) speed-of-sound [mm/ $\mu \mathrm{s}]$ using 16 views. Profiles through the center of the reconstructed images of (c) absorbed optical energy density and (d) speed-of-sound.

of both $\mathbf{A}$ and $\mathbf{c}$ can be obtained using only 16 views (Fig. 4). The results are shown after 36 iterations. The regularization parameter values $\lambda$ and $\mu$ were $10^{-4}$ and $10^{-3}$, respectively. The reconstructed SOS map using the USCT measurements alone was employed as the initial guess for the SOS.

\section{SUMMARY}

Joint reconstruction of SOS and absorbed optical energy density was performed using a combined dataset of both PACT and USCT measurements. This approach yielded more accurate reconstructed images when compared with joint reconstruction using PACT data alone. Further, by exploiting the acoustic information in the PACT measurements, accurate estimates of the SOS distribution were obtained using only 16 views for the USCT dataset. Additional views were needed to obtain similarly accurate estimates using the USCT data alone.

\section{ACKNOWLEDGMENTS}

Special thanks to Chao Huang for all his helpful advice. This work was supported in part by NIH awards CA167446 and EB01696301.

\section{REFERENCES}

[1] Duric, N., Littrup, P., Poulo, L., Babkin, A., Pevzner, R., Holsapple, E., Rama, O., and Glide, C., "Detection of breast cancer with ultrasound tomography: First results with the Computed Ultrasound Risk Evaluation (CURE) prototype," Medical Physics 34(2), 773 (2007).

[2] Duric, N., Littrup, P., Roy, O., Schmidt, S., Li, C., Bey-Knight, L., and Chen, X., "Breast imaging with ultrasound tomography: Initial results with SoftVue," in [ International Ultrasonics Symposium], 382-385, IEEE (July 2013).

[3] Ruiter, N. V., Gbel, G., Berger, L., Zapf, M., and Gemmeke, H., "Realization of an optimized 3d USCT," in [Proc. SPIE], D’hooge, J. and Doyley, M. M., eds., 796805-796805-8 (Mar. 2011).

[4] Wang, L. V., "Prospects of photoacoustic tomography," Medical Physics 35(12), 5758 (2008).

[5] Xia, J., Huang, C., Maslov, K., Anastasio, M. A., and Wang, L. V., "Enhancement of photoacoustic tomography by ultrasonic computed tomography based on optical excitation of elements of a full-ring transducer array," Optics letters 38(16), 3140-3143 (2013).

[6] Wang, L. V., "Multiscale photoacoustic microscopy and computed tomography," Nature Photonics 3, 503509 (Sept. 2009).

[7] Wang, L. V. and Hu, S., "Photoacoustic Tomography: In Vivo Imaging from Organelles to Organs," Science 335, 1458-1462 (Mar. 2012).

[8] Su, R., Ermilov, S. A., Liopo, A. V., and Oraevsky, A. A., "Three-dimensional optoacoustic imaging as a new noninvasive technique to study long-term biodistribution of optical contrast agents in small animal models," Journal of biomedical optics 17(10), 1015061-1015067 (2012). 
[9] Jose, J., Willemink, R. G. H., Steenbergen, W., Slump, C. H., Leeuwen, T. G. v., and Manohar, S., "Speedof-sound compensated photoacoustic tomography for accurate imaging," Medical Physics 39, 7262-7271 (Dec. 2012).

[10] Jiang, H., Yuan, Z., and Gu, X., "Spatially varying optical and acoustic property reconstruction using finite-element-based photoacoustic tomography," JOSA A 23(4), 878-888 (2006).

[11] Huang, C., Wang, K., Nie, L., Wang, L., and Anastasio, M., "Full-Wave Iterative Image Reconstruction in Photoacoustic Tomography With Acoustically Inhomogeneous Media," IEEE Transactions on Medical Imaging 32, 1097-1110 (June 2013).

[12] Beck, A. and Teboulle, M., "A Fast Iterative Shrinkage-Thresholding Algorithm for Linear Inverse Problems," SIAM Journal on Imaging Sciences 2, 183-202 (Jan. 2009).

[13] Huang, C., Wang, K., Schoonover, R. W., Wang, L. V., and Anastasio, M. A., "Practical limitations of joint reconstruction of absorbed optical energy density and speed of sound distributions in photoacoustic computed tomography," (2014).

[14] Stefanov, P. and Uhlmann, G., "Instability of the linearized problem in multiwave tomography of recovery both the source and the speed," Inverse Problems \& Imaging 7(4), 1367-1377 (2013).

[15] Krebs, J. R., Anderson, J. E., Hinkley, D., Neelamani, R., Lee, S., Baumstein, A., and Lacasse, M.-D., "Fast full-wavefield seismic inversion using encoded sources," Geophysics 74, WCC177-WCC188 (Nov. 2009).

[16] Moghaddam, P. P., Keers, H., Herrmann, F. J., and Mulder, W. A., "A new optimization approach for source-encoding full-waveform inversion," Geophysics 78, R125-R132 (Apr. 2013).

[17] van Leeuwen, T., Aravkin, A. Y., and Herrmann, F. J., "Seismic Waveform Inversion by Stochastic Optimization," International Journal of Geophysics 2011, 1-18 (2011).

[18] Zhang, Z., Huang, L., and Lin, Y., "Efficient implementation of ultrasound waveform tomography using source encoding," in [Proc. SPIE], 8320, 2 (Feb. 2012).

[19] Romero, L. A., Ghiglia, D. C., Ober, C. C., and Morton, S. A., "Phase encoding of shot records in prestack migration," Geophysics 65(2), 426-436 (2000).

[20] Wang, K., Matthews, T. P., Anis, F., Li, C., Duric, N., and Anastasio, M. A., "Waveform inversion with source encoding for breast sound speed reconstruction in ultrasound computed tomography," IEEE Transactions on Ultrasonics, Ferroelectrics and Frequency Control (in press).

[21] Plessix, R.-E., "A review of the adjoint-state method for computing the gradient of a functional with geophysical applications," Geophysical Journal International 167, 495-503 (Nov. 2006).

[22] Treeby, B. and Cox, B., "k-Wave: MATLAB toolbox for the simulation and reconstruction of photoacoustic wave-fields," Journal of Biomedical Optics 15(2), 021314 (2010).

[23] Tabei, M., Mast, T. D., and Waag, R. C., "A k-space method for coupled first-order acoustic propagation equations," The Journal of the Acoustical Society of America 111(1), 53 (2002).

[24] Mast, T. D., Souriau, L. P., Liu, D.-L., Tabei, M., Nachman, A. I., and Waag, R. C., "A k-space method for large-scale models of wave propagation in tissue," IEEE Transactions on Ultrasonics, Ferroelectrics and Frequency Control 48(2), 341-354 (2001). 